\title{
Balancing the quality of consent
}

\author{
Mats O Hansson Academic Hospital, Uppsala, Sweden
}

\begin{abstract}
The rule that one must obtain informed consent is well established in medical ethics and an intrinsic part of clinical practice and of research in biomedicine. However, there is a tendency that the rule today is being applied too rigidly and with too little sensitivity to the values that are at stake in connection with different kinds of research protocols. It is here argued that the quality of consent needs to be balanced against variables such as degree of confidentiality and importance of values at stake, in order to be ethically acceptable. Appropriate information and consent procedures should be adjusted accordingly. Three levels are suggested, ranging from extensively informed consent with both written and oral information, through informed refusal with only a limited amount of information given to, at the other end of the scale, just making relevant information available.
\end{abstract}

(Fournal of Medical Ethics 1998;24:182-187)

Keywords: Informed consent; informed refusal; Kant; utilitarianism

\section{Introduction}

In the recent Convention on Human Rights and Biomedicine drawn up by the Council of Europe it is stated in article 22 that: "When in the course of an intervention any part of a human body is removed, it may be stored and used for a purpose other than that for which it was removed, only if this is done in conformity with appropriate information and consent procedures".

However, it is not clear how the formula "appropriate information and consent procedures" should be interpreted in different cases. If taken in a too rigid sense much epidemiological research will in practice be precluded, with the consequence that new knowledge for the improvement of health and wellbeing may not be gained. Legal and ethical directions as to appropriate information and consent procedures interpeted too rigidly may actually be detrimental to the subjects that the convention on human rights seeks to protect. On the other hand, the potentiality that the information gathered about the individual may violate her or his integrity must not be underestimated. The difficulty of striking an ethically acceptable balance between different inter- ests is a challenge for all scientists active के $^{\circ}$ research which involves human beings, whether $\overrightarrow{\dot{m}}$ clinical and epidemiological research or in raindomised studies and research using computerisegd records.

The rule about obtaining informed consent well established in medical ethics and an intrinstc part of clinical practice and of biomedical and behavioural research. Its strong position as ethical rule in medicine since the Nuremberg toials is both understandable and legitimate. However, there is a tendency today for the rule to be applied too rigidly and with too little sensitivity the values that are at stake in connection with different kinds of research protocols. Medicineçs becoming more and more technically advanced and accordingly more difficult for people outsia the medical profession to understand. There is this situation a substantial risk that legislationais called for which is too rigid and not of benefit क़ेo anyone.

The rule about obtaining informed consent 1 been elaborated in detail in the medico-legal and medico-ethical literature concerning the meaning of its different components. These are: the competence of the research subject; that information is given in such a way that it can be funy understood; that a sufficient degree of voluntari ness is assured, and that authorisation is acquired from the research subject. ${ }^{2}$ To this list should be added a requirement for appropriate documentation of the information given and the conselit obtained. This is a requirement that needs muth more attention in medical research than it has tot received. What has not been discussed enoughnis the quality of consent that is required in different contexts. It is not reasonable that the rule about obtaining an informed consent should be same in situations of ordinary treatment, in clifyical trials and in protocols of epidemiologieal research where no personal identification is possible. The quality of consent needs to be balancerd against the different values that are at stake in different contexts. The kind of information, the it is given, the degree of voluntariness and the fermat of authorisation must be adjusted accordingly. I will here suggest a model for balancing the quality of consent against other variables tofat are ethically important in different contexss. 
According to the model I am suggesting, appropriate information and consent procedures vary depending on context between extensively informed consent with written and oral information through informed refusal with only a limited amount of information given, while at the other end of the scale it should just be a matter of making relevant information available.

\section{Values at stake and the priority of self-determination}

The primary values at stake in cases of ordinary medical treatment and in clinical trials are the integrity, the health and the wellbeing of the individual patient or research subject. In epidemiological research the primary value that must be respected is the integrity of the individual most directly concerned. Actions taken may also be of indirect importance for the integrity, the health and the wellbeing of other individuals, now or at a later time. In genetic medicine third parties are concerned in a special way since genetic information about an individual by definition also concerns genetic relatives of this individual. What is at issue in this context is not only the quality of consent but also the duty of professionals to inform genetic relatives of information that may be of interest to their health, wellbeing or integrity. However, I will not take up this special issue about the duty to inform here.

When the patient or the research subject is a minor or an individual with a low capacity for self-determination the informed consent must be obtained through a proxy decision by someone who acts in the best interest of the individual most directly concerned. However, this does not alter the need to balance the quality of that consent against other variables, just as is similarly done when fully competent persons are concerned.

The value of self-determination is the fundamental motivation of rules about obtaining informed consent. In the model presented here it is suggested that this value has to be balanced against other values and that other variables must be taken into consideration. However, the value of self-determination is given priority. The argument for this priority is Kantian in kind and may be elaborated briefly as follows. A minimal but fundamental requirement in morality is that a person must not be completely denied the possibility to be an agent and to be a member of a moral community. ${ }^{3}$ An acknowledgement of the right of self-determination is a necessary condition for a person to be a moral actor in this sense, and thus a fundamental and prior value for morality.
The priority of self-determination constitutes the demarcation line between moral and legal norms which is reflected in the two different kinds of sanctions associated with the two norm systems. Legal norms are associated with external sanctions. We can get a person to do something or to refrain from doing something by imposing external sanctions upon him. Moral norms, on the contrary, are entirely dependent on inner sanctions. We have to trust that a person has a fundamental capacity to experience moral shame. There must be something inside his mind which we can appeal to, namely, his capacity of self-determination, the most basic capacity of which is to assume moral responsibility. It is of course best if moral and legal norms work together but there must always be room for correcting and improving the law. However, this cannot be done merely through external sanctions. They would have no source. Morality is the source of and therefore prior to law, and self-determination is the necessary prior value to get morality off the ground.

To give priority to the value of selfdetermination is the minimal protection that is needed in order that a person shall be entitled to the status of moral maturity. However, this priority must not be applied too rigidly. When time is short, as in cases of emergency, or when no vital interests are endangered for the person directly concerned, self-determination should give way to other values. The quality of consent must be balanced accordingly.

\section{The factor of time and the ethics of emergency situations}

In contexts of emergency we expect doctors to adjust the quality of consent to the time that is available and to the severity of the condition of the patient(s). In these circumstances doctors adhere to strict medical criteria of urgency and a special ethics of triage is applied in order to select between different individuals who have been injured in a major accident. We also expect doctors to adjust the consent procedures in accordance with how serious the consequences of a medical action or treatment are. The greater the risk for damage, the more carefully elaborated we expect the information and consent process to be. Written information should be complemented with oral information, using personnel with good psychological and pedagogical skills, who are concerned with all aspects of the complex communication process. On the other hand, when estimated consequences to the individual most directly concerned are small, perhaps at the edge 
Figure 1 Balancing quality of consent in treatment and clinical research

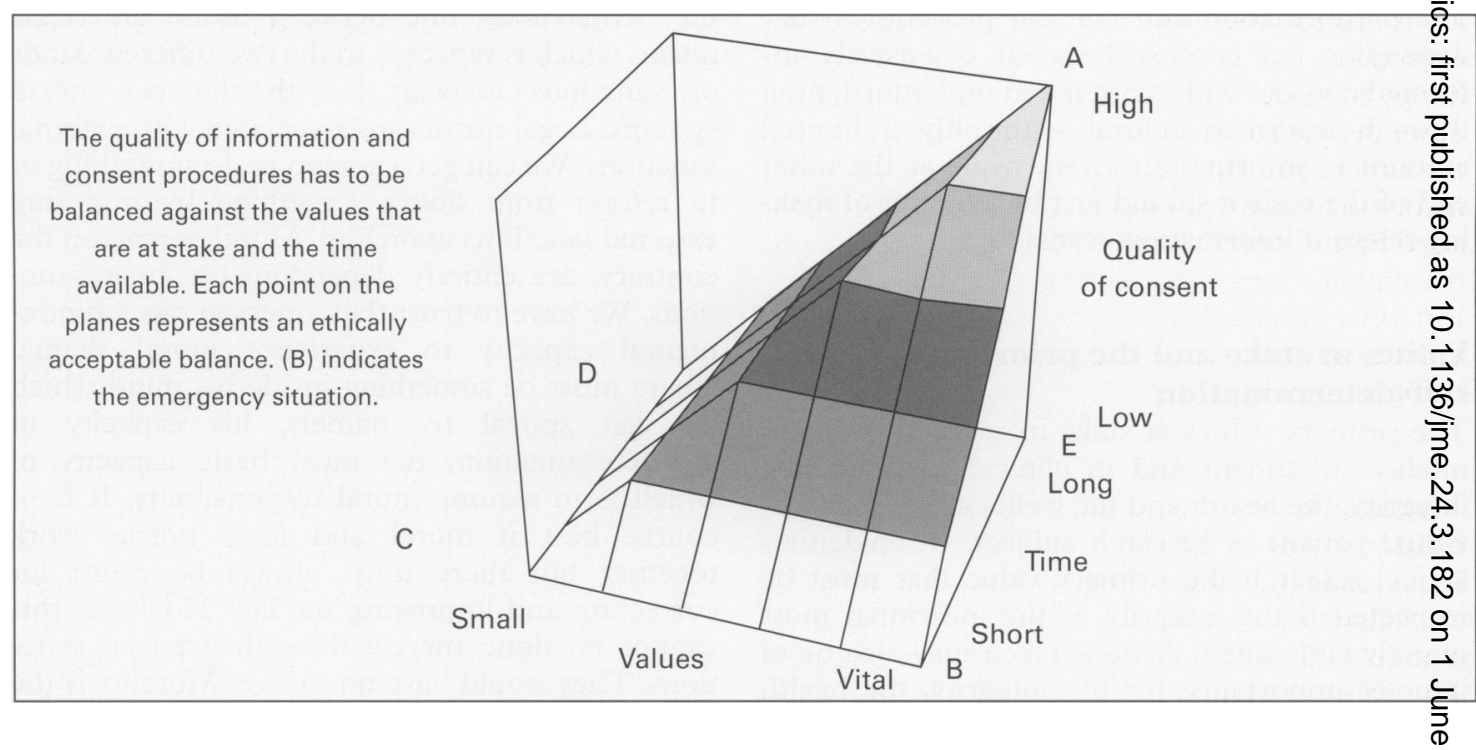

of triviality, we do not expect doctors to engage in long communication processes. This mode of balancing the quality of consent and the necessity to think simultaneously in several dimensions in ethical decision-making is illustrated in the above three-dimensional figure. Quality of consent is here balanced against the time that is available for the communication process and against the importance of the values that are at stake for the individual most directly concerned. The existence of an emergency ethics and the now established praxis of acting on the basis of a "presumed consent" in appropriate situations indicate that balancing of the quality of consent is already part of good medical and research practice.

The indicated three-dimensional inclined planes illustrate the ethical acceptability of decisions in ordinary treatment or in clinical trials. It should be observed that all decisions taking place on the planes are ethically acceptable. The planes are sloping but not slippery. It is possible to find a "stop-point" at any place on the planes. This model of ethical decision-making represents a soft deontological theory. It evades the problems associated with a rigid deontological theory which is insensitive to consequences. It is also dissociated from a utilitarian theory of the classical kind which is more easily willing to let selfdetermination give way to other values, even trivial ones. ${ }^{4}$

On these planes, A represents a decision where values at stake for the concerned patient are vital, time is long and, accordingly, appropriate information and consent procedures should be of high quality. Point B represents the emergency situation when great values are at stake but time is short. Points C and D represent decisions that ate morally uncontroversial since values are sms Point $\mathrm{E}$ is not morally permissible since sejfdetermination is given priority and there is time enough to obtain an informed consent. It should be observed that $\mathrm{E}$ would be allowed if a utilitarian theory of the classical kind had been chosengas the philosophical underpinning of this model $\mathrm{r}$ balancing the quality of consent. ${ }^{5}$

Values at stake for other individuals may, according to this model, not compete with vical values at stake for the individual most directly concerned according to a utilitarian calcuhs where all values always have to be subsumes under the best total outcome. This is the price thgat has to be paid for giving self-determination prigrity. However, as will be seen regarding epidemiological research, the comparative utility of different information and consent procedures has to $\mathrm{B}$ estimated and small infringements of persomal integrity allowed in order that new knowledge importance for other individuals' health and weidbeing may be gained.

It may be feared that self-determination oj secured at too high a cost and that important talues of health and wellbeing, which it is hoped be achieved through clinical research, will not $\mathscr{\text { e }}$ realised. However, the argument may easily be turned the other way around. It is an advantage 0 f the model that it provides individuals with opportunity to act on the basis of altruisic concerns. As moral actors they may want to act norms of unselfishness, solidarity or benevolenge, norms and attitudes that society has a strog g self-interest in encouraging. By giving sedfdetermination priority doctors are invited $\equiv$ 
conceive of their patients as collaborators. They are persons who, if fully informed about the objectives and means of the research, would be happy to make their contribution, to science or to other people suffering from disease. This is in fact the experience shared by many clinicians who are involved in clinical research. It must be emphasised that in order not to inflict undue pressure on the patient/research subject, and so deprive altruism of its moral value, a strict borderline has to be maintained between the two professional roles of being a doctor and being a researcher.

\section{The factor of confidentiality in epidemiological research}

In an analogous way we can now discuss the ethical balancing that takes place concerning information and consent in epidemiological research. The values at stake in this kind of research vary depending on the specific nature of each research protocol but can be subsumed as above under the general groups of integrity, health and wellbeing. Integrity is the most important value for the individual research subject. Indirectly, health and wellbeing may matter for this individual as well since she in the future also may benefit by new knowledge attained through epidemiological research. Epidemiological research is a necessary means for improving public health and minimising risk factors to peoples' health and prospects of wellbeing. It is important to strike a proper balance between the integrity of the individual and other benefits at stake for individuals, groups and society at large. In the model suggested here degree of confidentiality is the key element that is needed in order to find a proper balance between the quality of consent and the values at stake in epidemiological research. In this kind of research there is an interest in compiling information about individuals which is not directly relevant to their own health and wellbeing but which may be of vital interest to other individuals and society at large.

In some research protocols it is possible to achieve a confidentiality for the concerned individuals which is so strict that neither they nor their relatives will be able to be identified, either at the time or in the future. In other cases confidentiality may be attained for each individual but not for the group to which the individual belongs. If an ethnic group has been associated with a stigmatising genetic condition an individual risks discrimination just by being a member of the group. No further personal information is necessary. There are several cases in the history of medical research where individuals have been socially stigmatised even if there was no information available at the individual level. The integrity of the group, ethnic or other, was violated. Taking the recent development of genetic diagnosis into consideration the value of group integrity must be carefully assessed before protocols in molecular epidemiology can be ethically approved.

In some cases a blood or tissue sample may be taken as part of a routine measure without any specific research purpose being indicated when the sample is taken. However, in the future these samples may be of vital use for epidemiological research and there may arise an interest of joint processing of several different computerised records containing medical information about individuals. Because different values are at stake, appropriate consent procedures must not be the same in all these different cases. The quality of consent must be balanced against the values that are at stake for the individual directly concerned. The degree of confidentiality reflects the level of integrity that is threatened.

A special problem related to epidemiological studies where small values are at stake for the individuals most directly concerned is that if extensive information and consent procedures are required the response rate may drop. As a consequence the results of the study will be more uncertain and new vital knowledge may not be gained. Similar situations may occur in clinical research settings but in these contexts it is more likely that a good sense of partnership can be established between the researcher and the research subject. Response rates will be higher in accordance with the argument concerning altruism, referred to earlier. The personal contact between doctor and patient is important for encouraging altruistic attitudes to come into play. When there is only a small infringement of personal integrity or confidentiality can be assured, a comparative estimation of the utility in terms of response rates between different information and consent procedures is both an appropriate and ethically responsible consideration to make. With the parameters of quality of consent, vitality of values and degree of confidentiality we get the following three-dimensional plane for ethical balancing in epidemiological research. As in the previous case all points on the sloping planes represent ethically acceptable decisions.

Point A represents a decision when vital values (integrity, health and wellbeing) are at stake and confidentiality cannot be assured. In this case consent procedures have to be rigid. At point $B$ values may be vital but since strict confidentiality can be assured consent procedures may be lax. 
Figure 2 Balancing quality of consent in epidemiological research

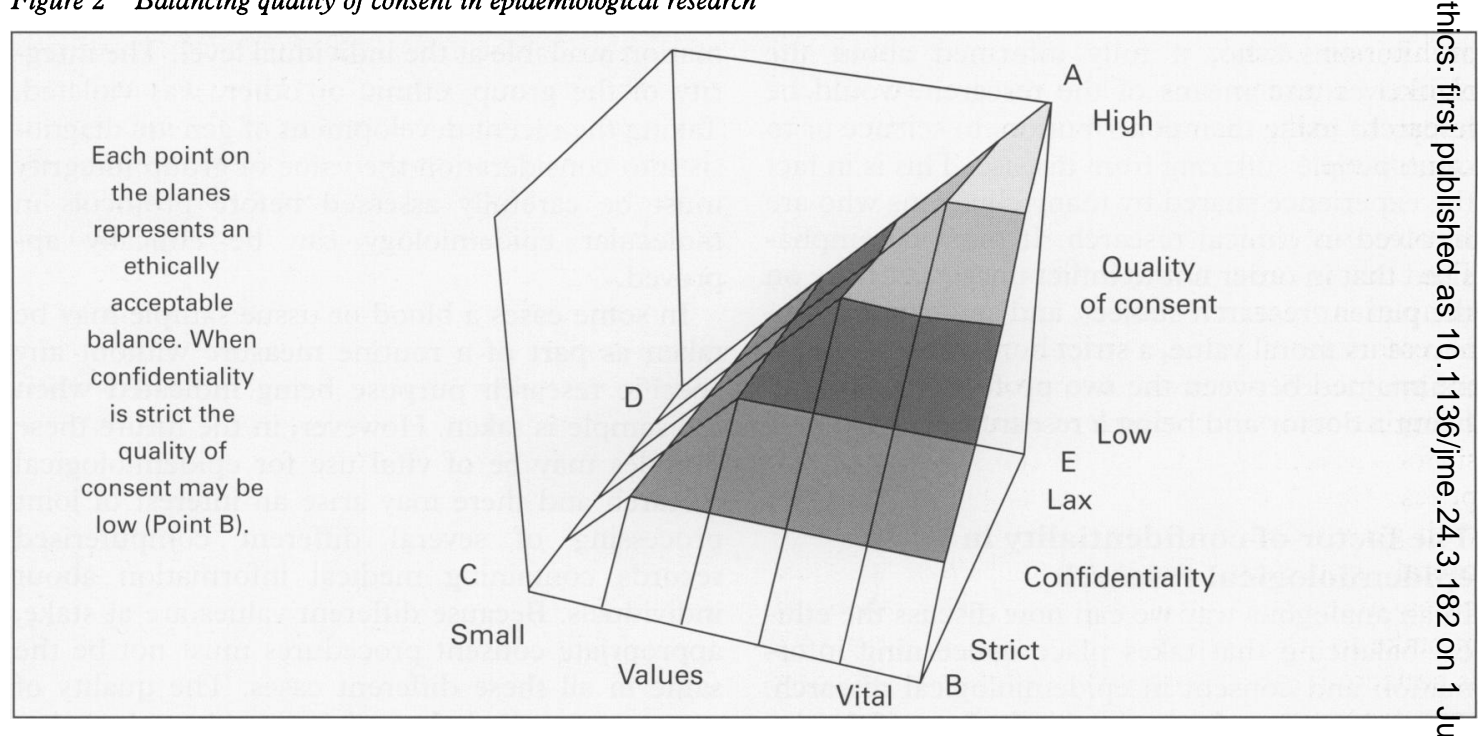

Points $\mathrm{C}$ and $\mathrm{D}$ are not morally controversial since values endangered are small. The quality of consent may be low. Point $\mathrm{E}$ is not morally permissible because of the priority given to self-determination.

\section{Appropriate information and consent procedures}

In drawing the concrete conclusions of these models, what should be considered appropriate information and consent procedures may vary along a continuum. The following three procedures may serve as heuristic steps for the professionals concerned with ensuring a quality of consent in specific situations that is ethically acceptable. The focus here is at the information element of the rule about obtaining an informed consent. If these information levels are acceptable careful elaboration of the details should be done by the professional bodies in collaboration with the relevant ethical committees. In this work due consideration should be given also to the other components, not least the requirement of documentation.

\section{EXTENSIVELY INFORMED CONSENT}

In most clinical trials as well as in ordinary treatment where great values are at stake for the individual directly concerned, it is appropriate to obtain an extensively informed consent with both written and oral information, and care must be taken to ensure that the obtaining of consent is a process, not a momentary thing. ${ }^{2}$

\section{INFORMED REFUSAL}

In cases where only small values are at stake for the individual most directly concerned it is appro- priate that only a limited amount of written inf $\stackrel{\text { or }}{\text { - }}$ mation be given. The individual should on trass basis be entitled to the right to refuse the planned intervention or collection of information. Therpe might, for example, be cases when epidemiologi-

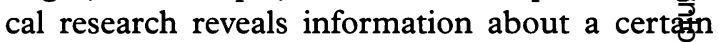
cohort but this information is not judged to $8 \mathrm{e}$ sensitive or socially stigmatising for any in\&ividual. However, the individual should have the benefit of the doubt concerning an estimation $=f$ the magnitude of the values at stake, and, accor ingly, a right to exercise an informed refusal. After this point has been made clear it should also $\overline{\mathrm{F}} \mathrm{e}$ emphasised that when it is beyond doubt that amy vital values are at stake for the individual directy concerned, this individual should not be entitligd the right to refuse information collection that is serving a vital, and therefore morally compelling, interest of other individuals or the public at large. Routine sampling of tissue or blood for future purposes is permissible according to the ethigal principles outlined in this paper, given the important proviso, however, that if it is estimated thet any future use might jeopardise vital values for this individual (integrity, health or wellbeing) then a procedure of informed refusal or extensive $y$ informed consent must be followed, depending on the context. In an analogous way routine registration of patients in a national medical register, for example a cancer register, is ethically permmssible. Later harm may be difficult to estimate b\&h for the individual most directly concerned and offspring. It is therefore important that future usse of stored samples and records is approved by⿳a⿴囗十⺝⿱ ethical committee. It should be observed thas a policy for appropriate information procedupes 
along these lines is in agreement with the common prohibition of asking research subjects to grant blanket consents for all future non-specified research using samples that are identified or identifiable. $^{6}$

\section{INFORMATION MADE AVAILABLE}

The taking and storage of tissue and blood samples is a part of ordinary medical practice and necessary for the diagnosis and monitoring of the the progress of a disease. A sample taken as part of a routine analysis or in order to confirm a diagnosis for a specific patient can be used for many purposes. New biochemical tests can be performed with great scientific value, something which is of moral significance for future patients. In these cases, under the provision that no vital values are at stake for the individual from whom the sample is collected, the appropriate information and consent procedure is to make known to patients that the taking of samples and their use for scientific purposes is part of standard procedure on any medical ward and that it is an important means for the improvement of future patients' health and wellbeing. It is an ethical requirement that information about this is given to patients and that more strict consent procedures are invoked as soon as any vital values for the directly concerned individual are threatened. The gravest $\sin$ in this context today is that often no information at all is made available to the patients concerning blood and tissue sampling. Good information procedures and good storage procedures must also be ensured in order that assurances about confidentiality shall be credible. A doctor who sends a sample for lab analysis does not "own" the sample and the sample can, for example by a researcher at the laboratory, be used for an additional analysis. ${ }^{7}$ However, good storage procedures are needed to ensure both that the potential of the sample is well managed and that scientific honesty between research colleagues prevails.

It might be objected that even if the argument for balancing the quality of consent is sound as it here stands, it doesn't help much as long as the values that are considered vital (integrity, health, wellbeing) do not have clear definitions. However, it is only after an argument for balancing has been accepted that there is a real need for conceptual clarification with regard to these values. Of primary importance is the need to investigate the notoriously difficult concept "integrity" and its role in biomedical research. It is often used as a self-evident ethical block but if other values are at stake detailed explanation is needed both concerning what exactly it is that is threatened and why it is considered to be violated in connection with a specific research protocol.

Mats $O$ Hansson, BA, ThM, ThD, is Associate Professor of Ethics in the Department of Public Health and Caring Sciences at the Academic Hospital, Uppsala, Sweden.

\section{References}

1 Council of Europe. Convention for the protection of human rights and dignity of the human being with regard to the application of biology and medicine: convention on human rights and biomedicine. Strasbourg, 1996 Nov 19.

2 For one detailed elaboration see Beauchamp TL, Childress JF. Principles of biomedical ethics [3rd ed]. Oxford: Oxford University Press, 1989: 67-113.

3 Concerning the moral importance of protecting agency see O'Neill O. Constructions of reasons. Explorations of Kant's practical philosophy. Cambridge: Cambridge University Press, 1989: 51-77.

4 For a critique of classical utilitarian theory concerning this deficiency see Parfit D. Reasons and persons. New York: Oxford University Press, 1984: 381-90.

5 Due to its requirement of maximising the total outcome, a utilitarian motivation of the model would allow all decisions represented by the volume under the two planes. In contrast to what Beauchamp and Childress believe (see reference 2), this shows that the selection of philosophical motivation does make a difference for the balancing of ethical principles in practice.

6 ASGH Report. Statement on informed consent for genetic research. American fournal of Human Genetics 1996;59:471-4.

7 The "ownership" of this kind of samples has been extensively discussed. In accordance with an argument by Swain and Marusyk they should be conceived as things held in common, in accordance with a Lockean perspective of ownership and property rights, they are things owned by no one but they may be used for the benefit of humanity. As soon as someone, for example a pharmaceutical company, has invested labour and so transferred the sample material into a commercial product they have acquired the property right of the product. See Swain MS, Marusyk RW. An alternative to property rights in human tissue. Hastings Center Report 1990;Sept/Oct: 12-15. 